\title{
Monotonic transition based forward body bias for dual threshold voltage low power embedded processors
}

\author{
S. Jayapal and Y. Manoli \\ Chair of Microelectronics, Department of Microsystems Engineering (IMTEK), University of Freiburg, \\ Georges-Koehler-Allee 102, 79110 Freiburg, Germany
}

\begin{abstract}
Dual threshold voltage and forward body bias techniques are effective ways to optimally balance the standby leakage power and performance. In this paper, we propose a novel fine-grained forward body biasing scheme for monotonic static logic circuits. In the proposed scheme, the forward body bias is applied to high threshold voltage of either the pull-up or the pull-down network based on the evaluation transition and the state of operation. This technique improves the low skew NAND and NOR circuit performance by $7 \%$ and $11 \%$, high skew NAND and NOR by $8 \%$ and $13 \%$ respectively. It reduces both active and standby leakage power as compared to monotonic static CMOS with dual- $V_{T}$ technique. The simulations are carried out using $130 \mathrm{~nm}$ mixed mode process technology to validate our proposed technique.
\end{abstract}

\section{Introduction}

In nanometer era, leakage power becomes gradually a major portion of the total power consumption in battery powered embedded systems. This increase in leakage power reduces the battery life time in such applications as wireless sensors with high standby to active time ratio (Jayapal et al., 2005a). To best utilize the battery source, both the active and standby leakage power has to be minimized while meeting the performance demands.

Modern CMOS process offers different types of threshold voltages and oxide thickness devices to meet the low power and high performance demands. The low and high threshold device provides high performance at the cost of high leakage and low leakage with low performance respectively. Depending on the application requirements, the designers can make optimal power performance trade-offs. For low standby power applications, dual threshold voltage with

Correspondence to: S. Jayapal (jsenthil@imtek.de) power gating reduces the standby leakage power at the cost of performance through traditional static CMOS logic circuits. On the other hand, in case of pass logic circuits an additional input gating is required at both ends of the customized pass logic blocks to reduce the standby leakage power (Jayapal et al., 2005b). To overcome this delay penalty and to reduce the transistor count, the high threshold voltage with forward body bias for improving the performance level has been introduced. The change in threshold voltage due to the bulk source potential is given by

$$
\Delta V_{t}=\gamma \sqrt{2 \Phi_{F}+V_{s b}}-\sqrt{2 \Phi_{F}}
$$

where $\gamma=$ body effect parameter, $\Phi_{F}=$ Fermi potential and $V_{s b}=$ SourceBulkpotential. Recent research reports indicate that the optimal forward body bias (FBB) value of 400 $500 \mathrm{mV}$ provides $13 \%$ and $37 \%$ performance improvement at $130 \mathrm{~nm}$ and $90 \mathrm{~nm}$ triple well process (Narenda etal., 2003; v. Arnim et al., 2004). This clearly suggests that the efficiency of body bias depends on process technology.

Monotonic Static CMOS circuits provide noise immunity low power and high performance (Solomatnikov et al., 2000), and differ from traditional static CMOS in the way transition are made. In order to have monotonic transitions at the gate outputs, the concept of pre-charge and evaluation mode resembles domino circuits. During the pre-charge mode, each gate output is either charged to $V_{D D}$ or discharged to $V_{S S}$. In the evaluation mode, the output either stays at its present value or makes a transition depending on the inputs.

The rest of the paper will discuss the limitation of global body bias, the essentials of transition based fine grained FBB and simulation results using monotonic static low and high skewed gates. 


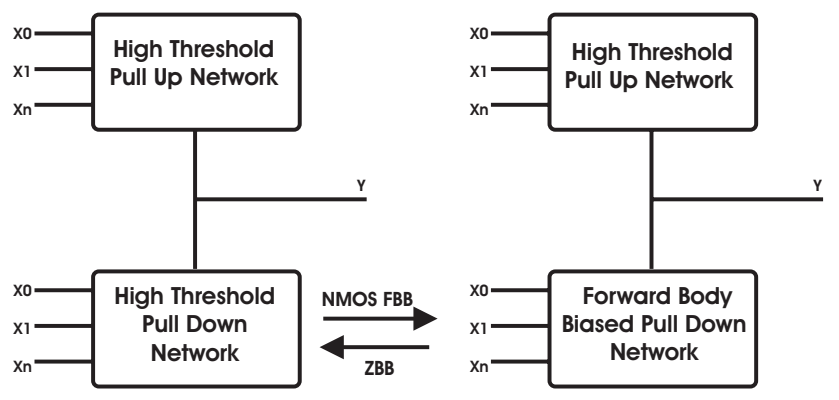

Fig. 1. Transition based fine grained forward body biasing with low skew NMOS body bias.

\section{Fine-grained forward body bias}

The application of global forward body bias using traditional static CMOS improves the performance at the expense of very high active leakage power. Applying forward body bias to high threshold device increases active leakage power from the non-evaluation transistors. On the other hand, it becomes challenging to place low and high threshold transistors in traditional static CMOS circuits depending on evaluation transitions. To overcome this, we chose monotonic static logic circuits in which placement of dual threshold devices based on evaluation transition becomes easier. However, this technique increases both active and standby leakage power because of static low threshold evaluation transistors. Similarly, applying FBB to the high threshold monotonic static CMOS logic causes more active leakage power similar to the monotonic static CMOS with dual- $V_{T}$ technique (Thorp et al., 1999). As a result, the increase in active leakage power makes global body bias inadequate for low standby power applications.

We propose a transition based fine-grained forward body bias using monotonic static CMOS to avoid unwanted active leakage with performance improvement as shown in Fig. 1. In the proposed scheme, body bias is applied to high threshold transistors of either the pull-up or the pull-down network depending on the evaluation transitions. In monotonic static circuits, the applied forward body bias increases the transition rate without resizing the evaluation transistors. During active mode, a chosen body bias of $450 \mathrm{mV}$ is applied to the evaluation transistors, which decreases the CV/I delay by increasing the $I_{o n}$. The non-evaluation pre-charge high threshold transistor offers lower active leakage power and the body biased low threshold evaluation transistor contributes performance enhancement. During standby mode, the applied Zero Body Bias (ZBB) brings the body biased evaluation transistor into high threshold state to reduce standby leakage power. Thus, the selective forward and zero body biased evaluation transistor reduces both active and standby leakage power using monotonic property.

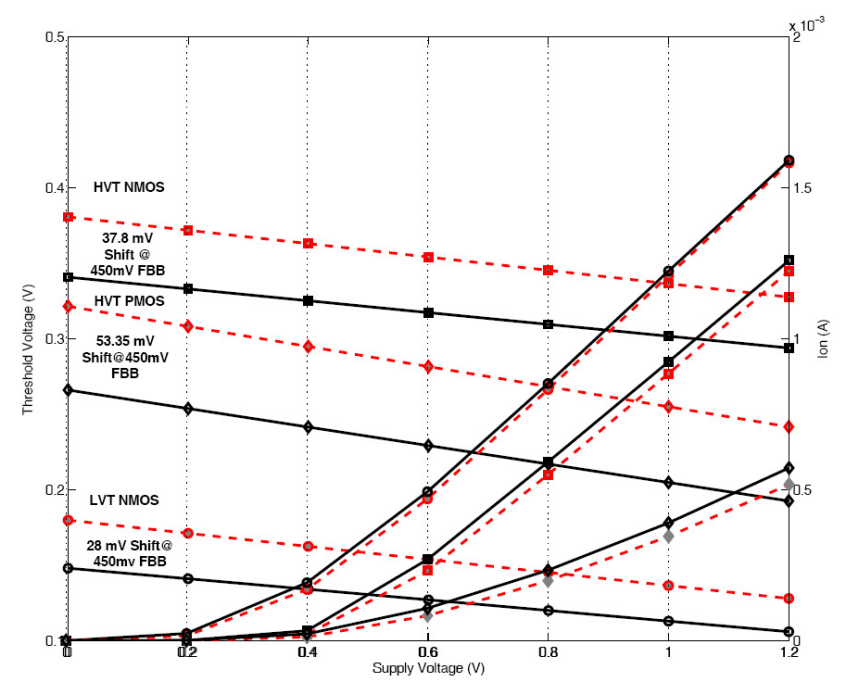

Fig. 2. The variation of threshold voltage and on-current of low and high threshold NMOS and PMOS devices.

Table 1. Threshold voltage variations and leakage currents for different supply voltages

\begin{tabular}{|c|c|c|c|c|}
\hline $\begin{array}{c}\text { Device } \\
\text { under } \\
\text { test }\end{array}$ & $\begin{array}{l}\Delta V_{T} \\
@ 1.2 \mathrm{~V} \\
\mathrm{VDD}\end{array}$ & $\begin{array}{c}\Delta V_{T} \\
@ 400 \mathrm{mV} \\
\mathrm{VDD}\end{array}$ & $\begin{array}{c}\triangle V I_{\mathrm{off}} \\
@ 1.2 \mathrm{~V} \\
\mathrm{VDD}\end{array}$ & $\begin{array}{c}\triangle V I_{\text {off }} \\
@ 400 \mathrm{mV} \\
\text { VDD }\end{array}$ \\
\hline $\begin{array}{c}\text { HVT } \\
\text { NMOS }\end{array}$ & $33.7 \mathrm{mV}$ & $37.8 \mathrm{mV}$ & $12.62 \mathrm{nA}$ & $41.4 \mathrm{nA}$ \\
\hline $\begin{array}{c}\text { LVT } \\
\text { NMOS }\end{array}$ & $22 \mathrm{mV}$ & $28 \mathrm{mV}$ & $0.54 \mathrm{uA}$ & $0.268 \mathrm{uA}$ \\
\hline
\end{tabular}

\section{Transition based forward body bias - comparison of power and performance}

Dual threshold $130 \mathrm{~nm}$ device measurement shows that applying $450 \mathrm{mV}$ body bias to the high threshold NMOS and PMOS increases $I_{\text {On }}$ by $3 \%$ and $11 \%$ at high drain source voltage. At the same time, the $I_{\text {on }}$ of low threshold NMOS are virtually identical due to severe Drain Induced Barrier Lowering (DIBL) and $V_{T}$ rolloff effects as shown in Fig. 2. Thus, the efficiency of body bias decreases with thinner oxide, shorter gate length and smaller $V_{T}$. Table 1 shows that the rate of change of threshold voltage with body bias at lower drain source voltage is greater due to reduced DIBL and $V_{T}$ rolloff. Dual $V_{T}$ high threshold PMOS measurement shows that the threshold voltage shift with $450 \mathrm{mV}$ FBB is about $1.4 \mathrm{x}$ more than those of NMOS devices. As a result, the larger $I_{o n}$ from body bias increases robustness of leakage 


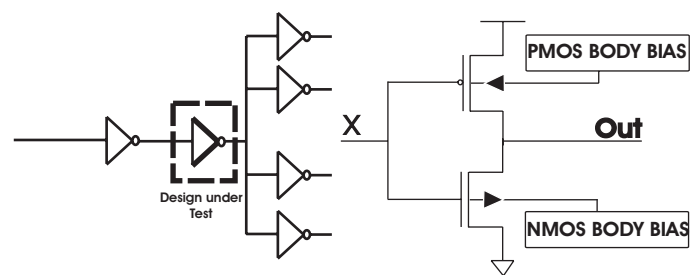

Fig. 3. Fan-out of 4 inverter delay with transition based forward body bias.

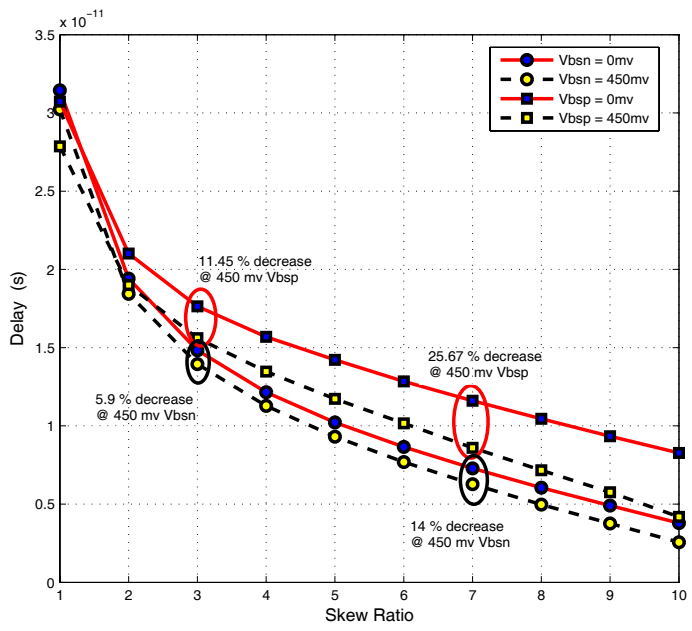

Fig. 4. FO4 Inverter delay variation with $450 \mathrm{mV}$ FBB for various skew ratios.

sensitive circuits such as domino logic and highly skewed static logic gates.

Lower operating temperature improves performance, however the leakage current increase due to $450 \mathrm{mV} F B B$ is about $3.2 \mathrm{x}$ and $4 \mathrm{x}$ at $1.2 \mathrm{~V}$ and $0.4 \mathrm{~V} V_{D S}$. Higher temperature offers $2.6 \mathrm{x}$ and $3.1 \mathrm{x}$ increase in leakage current at $1.2 \mathrm{~V}$ and $0.4 \mathrm{~V} V_{D S}$. Thus, the leakage current increase due to FBB at lower drain source voltage is slightly more than those of higher $V_{D S}$, irrespective of operating temperature.

To validate the proposed technique, the fan-out of 4 (FO4) inverter delay as shown in Fig. 3, is designed to compute the performance improvement for both evaluation transitions. Fig. 4 shows that the transition rate due to PMOS body bias has larger CV/I delay reduction compared to those of NMOS body bias. PMOS body bias provides the same CV/I delay with (1-2) $\mathrm{x}$ less skew ratio (the ratio between PMOS and NMOS transistor widths) compared to without body bias condition. On the other hand, the NMOS body bias offers the same transition rate with almost $1 \mathrm{x}$ less skew ratio.

\section{Monotonic static gates - design considerations}

Figure 5 shows the monotonic static CMOS logic gates, each gate is biased to make faster Low to High (L-H) or High to
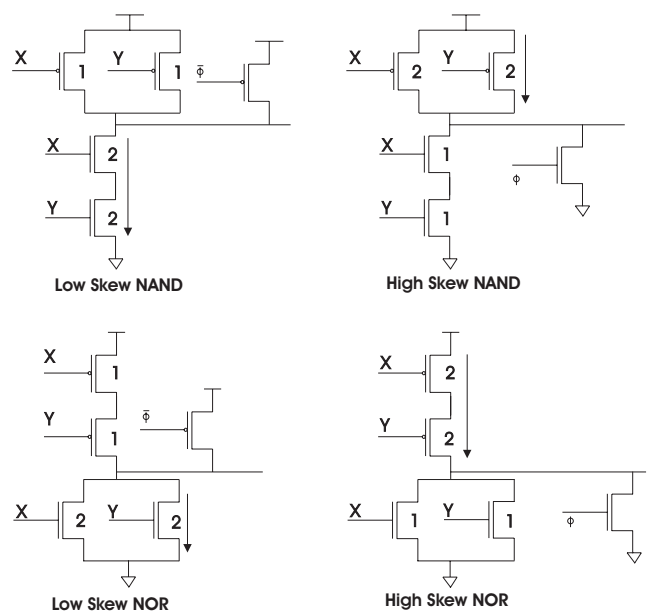

Fig. 5. Monotonic Static CMOS - Low and high skewed gates.

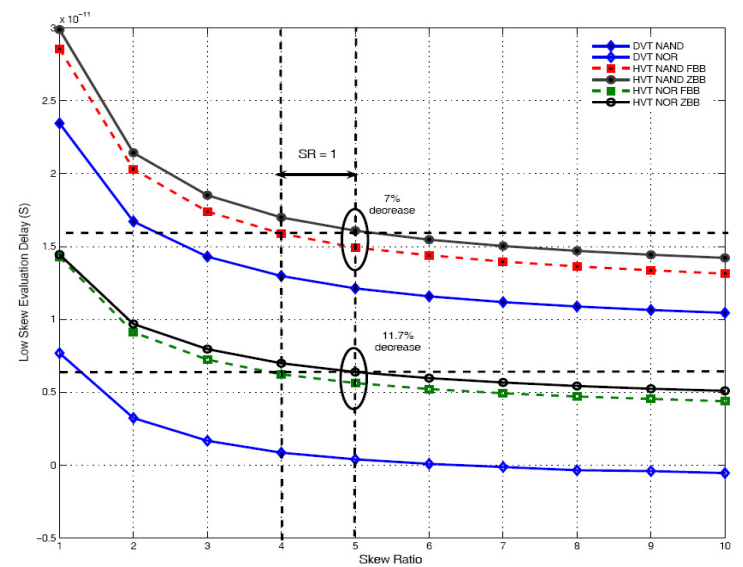

Fig. 6. Evaluation delay of low skewed gates for three different schemes.

Low (H-L) transitions. In general, there are two types of gates such as low skew (LS) gates which have faster H-L transitions and high skew (HS) gates with faster L-H transitions. Since monotonic logic is inherently non-inverting, it must be mapped to a network that does not contain intermediate inversions. This is in contrast to traditional static CMOS which can allow intermediate inverters. To utilize the monotonic property for fine-grained FBB, four different types of basic gates like LS-NAND, LS-NOR, HS-NAND and HSNOR were chosen. Each skewed gate is analyzed for three different schemes like high threshold with transition based forward body bias, high threshold without body bias and MSCMOS with dual $V_{T}$ (low $V_{T}$ in critical paths and high $V_{T}$ in non-critical paths) (Thorp et al., 1999) techniques.

Figures 6 and 7 show the evaluation delay and average switching power for LS-NAND and LS-NOR in three different techniques. Like FO4 inverter, the LS-NAND and LSNOR achieves the same CV/I delay with reduced skew ratio $(\mathrm{SR}=1)$. The parallel connected high fan-in LS-NOR gains 


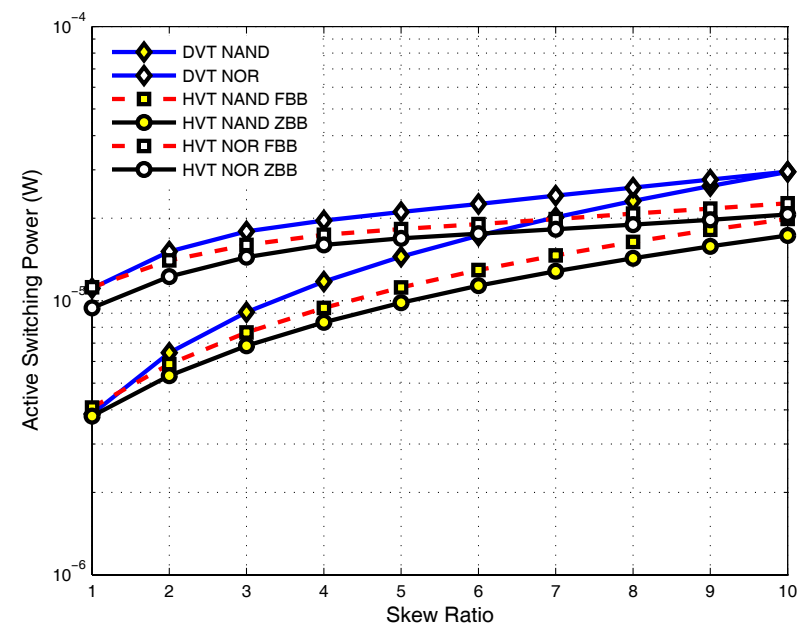

Fig. 7. Average switching power of low skewed gates for three different schemes.

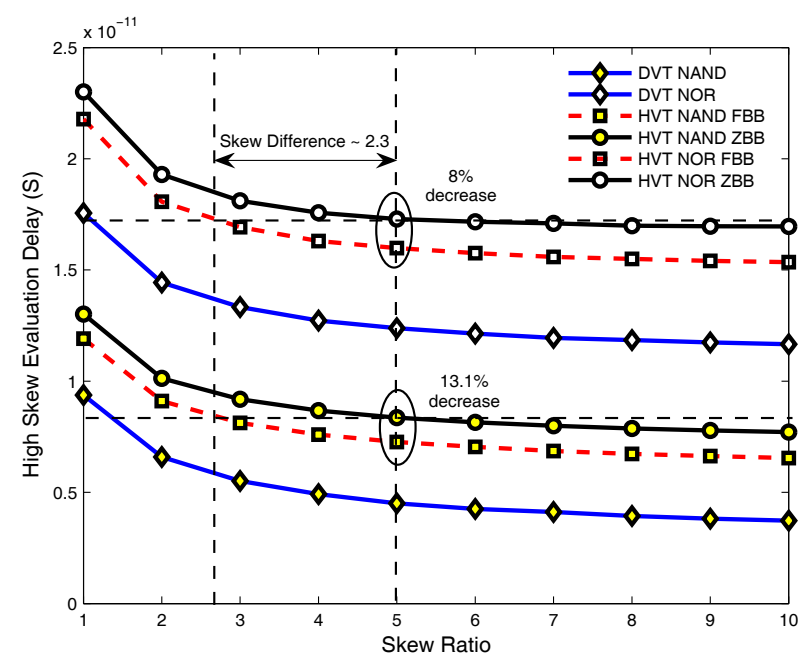

Fig. 8. Evaluation delay of high skewed gates for three different schemes.

0.67 times more delay reduction than the serial connected LS-NAND gate. The transition based body bias increases the switching power by $13.6 \%$ and $7.6 \%$ of LS-NAND and NOR gates, which is lower than that of MS-CMOS with dual $V_{T}$ techniques. Thus, the LS-NOR attains substantial performance gain with marginal increase in switching power using transition based body bias. On the other hand, the switching power increase due to body bias in LS-NAND is greater than that of LS-NOR gate.

Like low skewed gates, the large fan-in HS-NAND PMOS body bias reduces the evaluation delay by 0.6 times than those of HS-NOR gate as shown in Fig. 8. High skewed gates with PMOS body bias achieve the same CV/I delay at reduced skew ratio $(\mathrm{SR}=2.3)$. Thus, PMOS body bias gates achieve additional performance improvement with lower skew ratio than low skewed gates. From Fig. 9, the

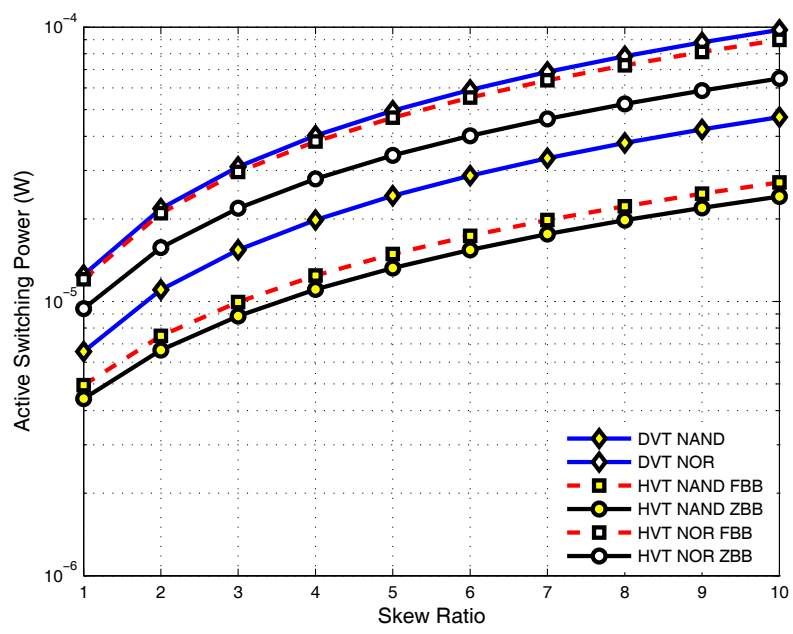

Fig. 9. Average switching power of high skewed gates for three different schemes.

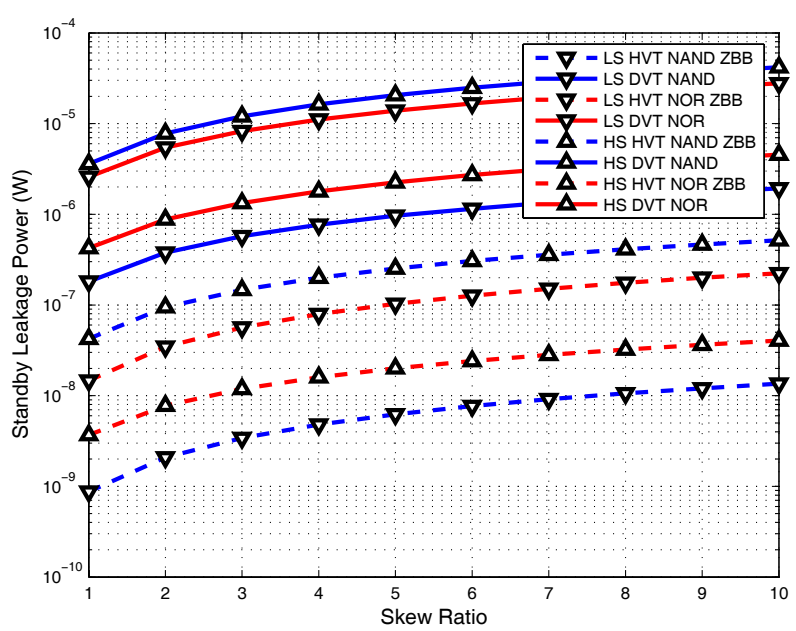

Fig. 10. The worst case standby leakage power of low and high skew gates.

average switching power of HS-NOR is increased by a factor of 2 when compared to HS-NAND. Transition based forward body biased high skew gate consumes lower switching power than the MS-CMOS with dual $V_{T}$ skewed gates.

The standby leakage power for both low and high skew gates are computed with worst case input patterns in Fig. 10. Applying Zero Body Bias (ZBB) during standby mode, brings the body biased skew gates to high $V_{T}$ state. In MS-CMOS with dual $V_{T}$ technique, the high dynamic and standby leakage power consumptions are mainly due to parallel connected low $V_{T}$ evaluation transistors. Series connected LS-NAND and HS-NOR consumes lower standby leakage power than that of parallel connected LS-NOR and HS-NAND gates. Thus, the worst case standby leakage power for the proposed scheme is far below the MS-CMOS with dual $V_{T}$ technique. 


\section{Conclusion}

Transition based fine grained forward body bias using monotonic property has been proposed. The proposed fine-grained body bias scheme provides the same circuit performance with reduced skew ratio for both low and high skew gates. This method consumes lower dynamic and standby leakage power than MS-CMOS with dual $V_{T}$ technique.

\section{References}

v. Arnim, K., Borinski, E., Seegebrecht, P., Fiedler, H., Brederlow, R., Thewes, R., Berthold, J., and Pacha, C.: Efficiency of Body Biasing in $90 \mathrm{~nm}$ CMOS for Low Power Digital Circuits, ESSCIRC, 175-178, 2004.

Jayapal, S., Huang, R., Ramachandran, S., Bhutada, R., and
Manoli, Y.: Optimization of Electr. Power Consumption in Wireless Sensor Nodes, 8th Euromicro conf. on Digital Syst. Design (DSD), 165-169, 2005a.

Jayapal, S., Sudalaiyandi, S., and Manoli, Y.: Efficiency of Leakage Reduction on Different Static Logic Styles for Embedded Applications with High Standby to Active Ratio, Int. Symp. on System-on-Chip (SoC), 151-154, 2005 b.

Narendra, S., Keshavarzi, A., Bloechel, B., Borkar, S., and De, V.: Forward body bias for microprocessors in $130 \mathrm{~nm}$ technology generation and beyond, IEEE J. Solid-State Circuits, 38, 696701, 2003.

Solomatnikov, A., Somasekhar, D., Roy, K., and Koh, C.-K.: Skewed CMOS: Noise-Immune High-Performance Low-Power Static Circuit Family, Int. Conf. Comp. Design, 241-246, 2000.

Thorp, T., Yee, G., and Sechen, C.: Monotonic static CMOS and dual Vt technology, IEEE/ACM Int. Symp. on Low Power Electronics and Design, 151-155, 1999. 